\title{
Strong genetic influence on hypocone expression of permanent maxillary molars in South Australian twins
}

\author{
Denice Higgins*, Toby E. Hughes, Helen James, Grant C. Townsend \\ Craniofacial Biology Research Group, School of Dentistry, The University of Adelaide, South Australia 5005
}

\begin{abstract}
An understanding of the role of genetic influences on dental traits is important in the areas of forensic odontology, human evolution and population variation. The aims of this study were: to calculate the frequency of occurrence and degree of expression of hypocones on permanent maxillary first and second molars in a sample of South Australian twins; to compare trait expression between males and females; to compare concordance rates for trait expression between monozygotic (MZ) and dizygotic (DZ) twins; and to fit genetic models to the data derived from twins and determine heritability estimates. Using stone dental casts, hypocone expression was scored on maxillary permanent first and second molars of $45 \mathrm{MZ}$ twin pairs and $43 \mathrm{DZ}$ pairs. Degrees of expression were scored from absence, through minor wrinkles or ridges, to very large cusps (score $0-5$ ) using the standardized method of Turner et al. (1991). Hypocones were found to be more common and larger on first molars than second molars and there was a tendency for them to
\end{abstract}

A central focus of dental anthropological study over the last century or so has involved metric and nonmetric analysis of the features of human teeth (Scott and Turner, 1977). The crowns of upper molar teeth have four main cusps and these are termed the paracone (mesiobuccal), protocone (mesiolingual), metacone (distobuccal), and hypocone (distolingual). Hypocone expression, like other non-metric dental crown traits, is generally scored by comparison with standardised plaques (Turner et al., 2001). These plaques aid visual assessment of presence and degree of expression. In the context of phylogeny, dental characters are associated with functional demands and dietary adaptations but also reflect the developmental processes controlling morphogenesis.

The field theory that was proposed by Butler (1939) and adapted by Dahlberg (1945) in an attempt to account for the common features of teeth within a class, postulated that the most mesial tooth in each morphological class is usually the most stable phenotypically. Osborn (1978), in his clone theory, proposed that a single clone of preprogrammed cells led to the development of all teeth within a particular class. Both of these theories provide be larger in males although this was not statistically significant. No significant differences in occurrence or expression were noted between antimeres, with fewer differences observed between first than second molars. The percentage concordance for expression in MZ twin pairs was higher than in DZ twin pairs indicating a genetic influence determining the variation observed in hypocone expression. The most parsimonious model to explain observed variation was an AE model, incorporating additivegenetic and uniqueenvironmental effects. Narrow-sense heritability estimates for both the first and second molars were high indicating that a large portion of the phenotypic variation could be explained by additive genetic effects. The greater range of phenotypic expression shown by the second molars compared with the first molars may reflect a common genetic liability that is modulated by differences in tooth size, location and/or developmental timing between these teeth. Dental Anthropology 2009;22(1):1-7.

insights into the mechanisms that may be involved in patterning within the human dentition. Recent progress in studying these mechanisms at a molecular level indicates the involvement of homeobox-containing genes (Mitsiadis and Smith, 2006). Recently, Mitsiadis and Smith (2006) and Townsend et al. (2008) have proposed a new genetic developmental model for teeth that incorporates the field, clone and homeobox code theories.

Current evidence on development shows that tooth morphogenesis is punctuated by transient signaling centers in the epithelium, the primary and secondary enamel knots, corresponding to the initiation of tooth crowns and individual cusps (Jernvall, 2000). Differential growth and subsequent folding of the dental epithelium is directed by the enamel knots, which are composed of non-dividing cells. Cell proliferation around the

*Correspondence to: Dr Denice Higgins, Forensic Odontology Unit, School of Dentistry, Faculty of Health Sciences, University of Adelaide, 5005, South Australia. E-mail: denice.higgins@adelaide.edu.au 
enamel knots is believed to be influenced by members of the fibroblast growth factor family. Genes involved in cusp development appear to be the same among all the individual cusps, with no particular gene for a single cusp, which means that at the level of molecular signaling, all the cusps are alike. A patterned cascade mode of cusp spacing may promote the evolution of new cusps and individual teeth may differ only in the timing of cusp initiation (Jernvall, 2000). As the secondary enamel knot program is repeated for every cusp, any small difference in cusp spacing will have a cumulative effect on later-developing cusps (Jernvall, 2000). Reflecting this concept, studies have shown that hypocones show the greatest variation in size of all upper maxillary molar cusps in hominoid primates and in humans (Jernvall and Jung, 2000; Kondo et al., 2005).

Insight into the relative contributions of genetic and environmental factors to human tooth development can be gained from twin studies involving the comparison of monozygotic (MZ) and dizygotic (DZ) twin pairs. Differences between MZ twin pairs can be expected to be of similar magnitude to the minor left right differences that may be observed in singletons, whereas the differences between DZ pairs are similar to those seen in siblings (Kabban et al., 2001).

The purpose of this study was to investigate size variability of the hypocone of permanent maxillary first and second molars in a sample of South Australian twins. The specific hypotheses that were tested were:

- That hypocones occur more frequently and are larger in first molars compared with second molars

- That hypocones occur more frequently and are larger in males than females

- That hypocone expression is symmetrical between antimeric teeth

- That monozygotic twin pairs exhibit a higher degree of concordance for hypocone trait expression than dizygotic twin pairs, indicating a genetic contribution to observed variation.

\section{MATERIALS AND METHODS}

From a collection of dental casts of over 600 twin pairs, $45 \mathrm{MZ}$ and $43 \mathrm{DZ}$ pairs were selected. The twins were all of European ancestry and were aged between 10 and 46 years. Only individuals with permanent maxillary first and second molars present on both left and right sides were included. Subjects selected did not have any extensive restorations and their casts were not damaged. The study was approved by the Committee on the Ethics of Human Experimentation, University of Adelaide (Approval No. H/07/84) as part of an ongoing study of the teeth of Australian twins.

Hypocones were scored on right and left maxillary first and second molars using Turner's ASU classification system (Arizona State University System, Plaque 8) (Scott and Turner, 1997) with 6 grades of expression.
Score 0 represented absence of a cusp, score 1 indicated a ridge or wrinkle present at the cusp site, score 2 was a faint cuspule, score 3 was a small cusp, score 4 was a large cusp and score 5 was a very large cusp. The casts were examined under a magnifying light and the degree of expression was determined by reference to a plaster replica of the scoring plaque. Assessments were made for all subjects on two separate occasions so that concordance rates between determinations could be calculated. A second observer scored 30 randomly selected casts for determination of inter-examiner reliability.

Statistical analysis was carried out using SPSS for Windows ${ }^{\odot}$. Frequencies were calculated for right and left side teeth, and for males and females. Associations between sides, first and second molars, and sexes were tested using chi-square tests. Statistical significance was set at an alpha of 0.05 .

As a preliminary assessment of possible genetic influence on phenotypic expression, concordances rates were calculated for MZ and DZ pairs for all hypocone expressions. Structural equation modelling was then undertaken using the software package $\mathrm{Mx}$ (Neale et al., 2006). Mx is a structural equation modelling package, flexible enough to fit a variety of mathematical applications. At its heart is a matrix algebra processor. There are many built-in fit functions to enable structural equation modelling (SEM) and other experiments in matrix algebra and statistical modelling, including facilities for maximum likelihood estimation of parameters from missing data structures, under normal theory. Complex 'non-standard' models are easy to specify. For further general applicability, it allows users to define their own fit functions, and optimization may be performed subject to linear and nonlinear equality or boundary constraints.

Mx can be used to apply structural equation models to variance-covariance matrices derived from monozygotic (MZ) and dizygotic (DZ) twin data. This method is particularly well-suited for continuously distributed data. However, SEM methodology can be extended to dichotomous and ordinal twin data by substituting the tetrachoric or polychoric correlation matrix (Pearson, 1901) for the variance-covariance matrix (Neale and Cardon, 1992). Use of SEM methodology for ordinal data is dependent on the assumption of an underlying continuous liability distribution that is bivariate normal. That is, it is assumed that categories are formed by imposing thresholds on a continuous liability distribution (Falconer, 1965; Reich et al., 1972).

Four sources of variation: $\mathrm{A}$, additive genetic variance; $\mathrm{D}$, non-additive genetic variance; $\mathrm{C}$, common [shared] environmental variance; and $\mathrm{E}$, unique [nonshared] environmental variance were modelled for twin pairs. A represents the additive effects of the alleles at a locus, whilst D refers to intralocus gene interactions. 
C affects twin similarity regardless of zygosity, whereas $E$ only represents unique effects contributing to withinpair differences.

Implicit in the model-fitting were the normal assumptions of the twin method: that mating was random; that trait-related, shared environmental influences on $\mathrm{MZ}$ and $\mathrm{DZ}$ twins were equal; and that there was no GxE interaction or gene-environment covariation (Jinks and Fulker, 1970). Since fitting models with four parameters to data from a classical twin study (MZ and DZ twins reared together) results in an underidentified model (Grayson, 1989; Hewitt, 1989; Dempsey et al., 1999), subsets of three or fewer parameters were chosen.

Rectangular files of raw ordinal data were prepared as described by Neale et al. (2006) and utilized directly for univariate analyses of ordinal data, maximising the likelihood under a bivariate normal distribution model. For right and left first molars, scores of 3 or less were combined into a single category (i.e., $\leq 3$ ) as only one tooth was scored less than a 3. Starting values for model thresholds were estimated from raw frequencies.

When analysing raw data, there is no direct measure of goodness of model fit. Instead, nested sub-models can be compared by examining the log of the likelihood function $(\log \mathrm{L})$. Nested model differences in $-2 \log \mathrm{L}$ are distributed asymptotically as a $\chi^{2}$, with degrees of freedom equal to the differences in free parameters between nested sub-models (e.g., ACE vs AE $=1 \mathrm{df}$ ). Initially, a Cholesky decomposition of the data was undertaken to produce a saturated model fit against which to test goodness-of-fit of nested sub-models. Where models were not nested (i.e., ACE vs ADE), the relative magnitude of the log of the likelihood was used to indicate the parsimony of each model. The general approach was that of accepting a more complex model only when a simpler one had failed. Path coefficients (a, c, e) were estimated. Heterogeneity of causes of variation between sexes was also evaluated.

Narrow-sense heritability estimates $\left(h^{2}\right)$ were calculated from the ratio of genetic variation (A) to total phenotypic variation $(\mathrm{A}+\mathrm{C}+\mathrm{D}+\mathrm{E})$ in the best-fitting model. Values of heritability estimates near 1 indicate that most of the phenotypic variation can be explained by additive genetic effects whereas values near zero indicate that environmental effects account for most of the variation in the phenotype.

\section{RESULTS}

Concordance between the first and second sets of scores was $98 \%$ and there was no indication of systematic methodological errors. Inter-examiner concordance was $72 \%$ and the discrepancies found were generally of the order of plus or minus one category.

Hypocones were present on all permanent first molars and on a high proportion of second molars as demonstrated in Table 1. Pronounced expressions of hypocones were noted on first molars, with only one individual having a score of less than 3, and a high proportion of score 4 or 5 . The second molars demonstrated more variation in hypocone expression. Subsequent genetic analysis treated hypocone expression on first molars as an ordinal trait with fewer categories than the second molars, yielding significantly lower power than the model for second molars, and consequently broader confidence intervals for parameter estimates.

Females had more pronounced expressions of score 4 and 5 on first molars, whereas the second molars showed more variability. Only $2 \%$ of females showed scores of

TABLE 1. Expression of hypocone trait in males and females (one member of each twin pair) ${ }^{1}$

\begin{tabular}{|c|c|c|c|c|c|c|c|c|c|c|c|c|c|c|c|c|}
\hline \multirow[b]{4}{*}{ Score } & \multicolumn{8}{|c|}{ Males } & \multicolumn{8}{|c|}{ Females } \\
\hline & \multicolumn{4}{|c|}{ First molar } & \multicolumn{4}{|c|}{ Second molar } & \multicolumn{4}{|c|}{ First molar } & \multicolumn{4}{|c|}{ Second molar } \\
\hline & \multicolumn{2}{|c|}{ Right } & \multicolumn{2}{|c|}{ Left } & \multicolumn{2}{|c|}{ Right } & \multicolumn{2}{|c|}{ Left } & \multicolumn{2}{|c|}{ Right } & \multicolumn{2}{|c|}{ Left } & \multicolumn{2}{|c|}{ Right } & \multicolumn{2}{|c|}{ Left } \\
\hline & $\mathrm{n}$ & $\%$ & $\mathrm{n}$ & $\%$ & $\mathrm{n}$ & $\%$ & $\mathrm{n}$ & $\%$ & $\mathrm{n}$ & $\%$ & $\mathrm{n}$ & $\%$ & $\mathrm{n}$ & $\%$ & $\mathrm{n}$ & $\%$ \\
\hline 0 & 0 & 0.0 & 0 & 0.0 & 4 & 11.0 & 3 & 8.0 & 0 & 0.0 & 0 & 0.0 & 5 & 9.6 & 9 & 17.3 \\
\hline 1 & 0 & 0.0 & 0 & 0.0 & 8 & 22.0 & 5 & 14.0 & 1 & 1.9 & 0 & 0.0 & 15 & 28.8 & 8 & 15.4 \\
\hline 2 & 0 & 0.0 & 0 & 0.0 & 4 & 11.0 & 4 & 11.0 & 0 & 0.0 & 0 & 0.0 & 6 & 11.5 & 7 & 13.5 \\
\hline 3 & 0 & 0.0 & 0 & 0.0 & 9 & 25.0 & 12 & 33.0 & 3 & 5.8 & 3 & 5.8 & 15 & 28.5 & 17 & 32.7 \\
\hline 4 & 19 & 53.0 & 19 & 53.0 & 9 & 25.0 & 10 & 28.0 & 28 & 53.8 & 29 & 55.8 & 10 & 19.2 & 10 & 19.2 \\
\hline 5 & 17 & 47.0 & 17 & 47.0 & 2 & 6.0 & 2 & 6.0 & 20 & 38.5 & 20 & 38.5 & 1 & 1.9 & 1 & 1.9 \\
\hline
\end{tabular}

${ }^{1} \mathrm{n}=88$ 


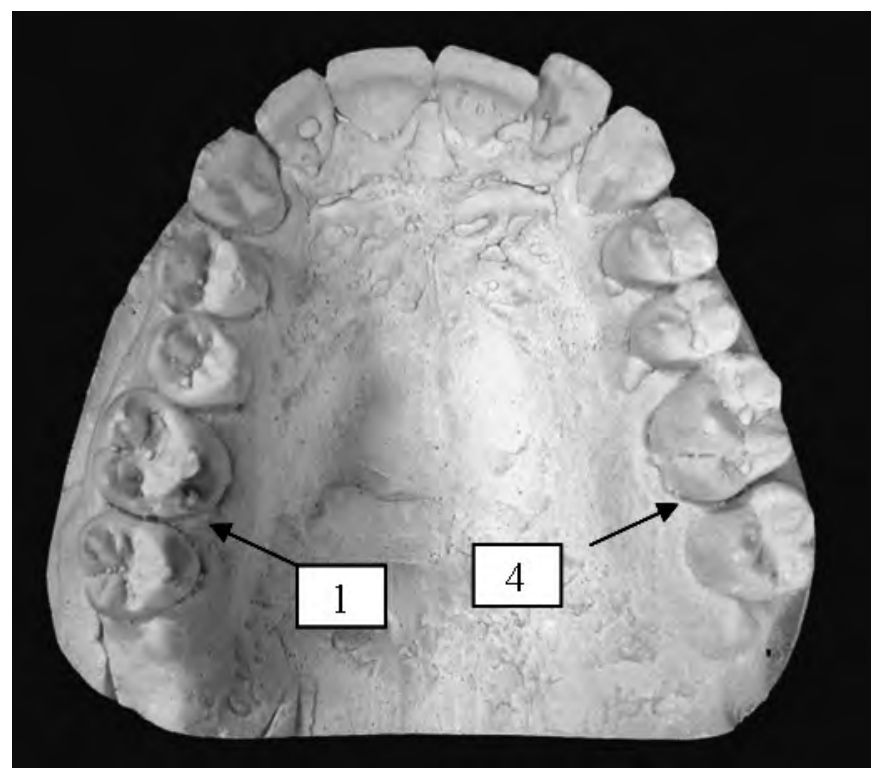

Fig. 1. Asymmetrical expression of hypocone trait on antimeric first molars - score 1 compared to score 4.

5 on both upper right and left second molars. Males showed a higher percentage of score 5 than females, with $47 \%$ of males showing score 5 compared to $38 \%$ of females. Females showed a higher percentage of score 4 and below. There was a tendency for male hypocones to be larger but this was not statistically significant.

In first molars, 98\% concordance in expression between antimeric teeth was noted. The only example of marked asymmetry was one individual with score 1 on the left first molar and score 4 on the right first molar, as shown in Figure 1. In second molars, the concordance

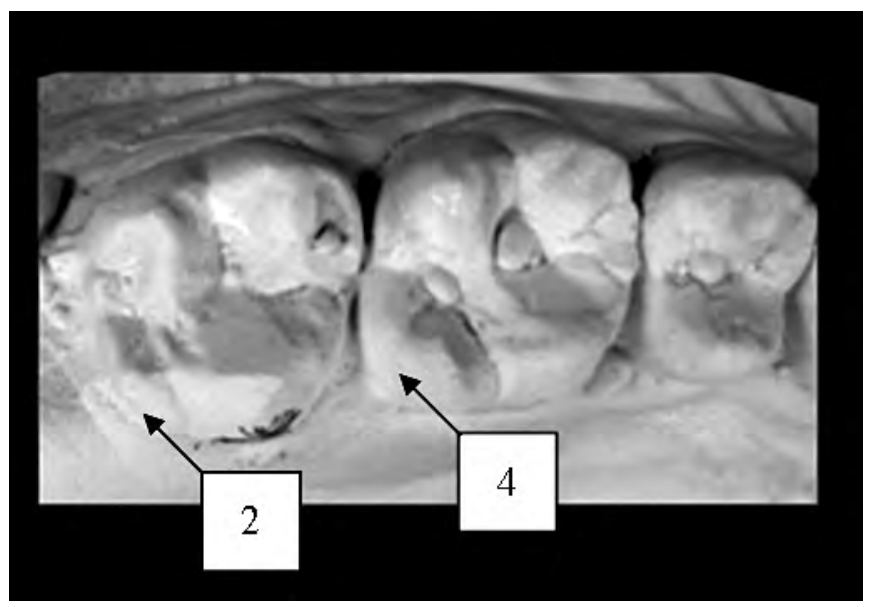

Fig. 2. Example of reduction in hypocone size from first to second molars - score 4 to score 2 .

rate for antimeres was $74 \%$.

The hypocone expression of first molars compared with second molars was examined in 88 individuals. One member from each of the 88 twin pairs (i.e. Twin A) was included in this analysis. As seen in Table 2, almost all of the scores for the maxillary right first molar were larger than those for the right second molar, except for five subjects - three had a score of 5 on both first and second molars, one had a score of 4 on the first molar and score 4 on the second, and one had score 3 on the first molar and score 4 on the second. When examining the maxillary left molars, again, most of the scores on the first molar were larger than those on the second molar except for four individuals - three had a score of 5 on both first and second molars and one had a score of

TABLE 2: Expression of hypocone trait on maxillary right first and second molars of individuals (one member of each twin pair $)^{1}$

\begin{tabular}{|c|c|c|c|c|c|c|c|c|}
\hline \multicolumn{9}{|c|}{ First molars } \\
\hline & Score & 0 & 1 & 2 & 3 & 4 & 5 & Total \\
\hline \multirow{7}{*}{$\begin{array}{c}\text { Second } \\
\text { molars }\end{array}$} & 0 & 0 & 1 & 0 & 1 & 5 & 2 & 9 \\
\hline & 1 & 0 & 0 & 0 & 1 & 17 & 5 & 23 \\
\hline & 2 & 0 & 0 & 0 & 0 & 8 & 2 & 10 \\
\hline & 3 & 0 & 0 & 0 & 0 & 16 & 8 & 24 \\
\hline & 4 & 0 & 0 & 0 & 1 & 1 & 17 & 19 \\
\hline & 5 & 0 & 0 & 0 & 0 & 0 & 3 & 3 \\
\hline & Total & 0 & 1 & 0 & 3 & 47 & 37 & 88 \\
\hline
\end{tabular}

${ }^{1} \mathrm{n}=88$ 
TABLE 3. Percentage concordance observed for hypocone trait expression in $\mathrm{DZ}$ and $M Z$ twin pairs

\begin{tabular}{lcccc}
\hline Tooth & $\begin{array}{c}\text { Right } \\
\text { M1 }\end{array}$ & $\begin{array}{c}\text { Right } \\
\text { M2 }\end{array}$ & $\begin{array}{l}\text { Left } \\
\text { M1 }\end{array}$ & $\begin{array}{l}\text { Left } \\
\text { M2 }\end{array}$ \\
\hline DZ & $67.4 \%$ & $25.6 \%$ & $67.4 \%$ & $18.6 \%$ \\
MZ & $80.0 \%$ & $62.2 \%$ & $80.0 \%$ & $68.9 \%$ \\
\hline
\end{tabular}

4 on both first and second molars. A typical example of reduction in cusp size from the first to second molar is shown in Figure 2.

MZ twin pairs exhibited a higher concordance rate for corresponding tooth comparisons than DZ twin pairs, as shown in Table 3. Percentage concordance for the first molars between MZ twin pairs was $80 \%$ whilst that for DZ twin pairs was $67 \%$. The concordance rate noted for the first molars was higher than that for the second molars, with the percentage concordance for the second molars being $65 \%$ for $\mathrm{MZ}$ twin pairs and $22 \%$ for DZ twin pairs.

An AE model was the most parsimonious for all variables. There was no significant heterogeneity between sexes for variance components for hypocone score. The final models represent pooled data from both sexes. As an example, Table 4 presents the pooled model structure and statistics for hypocone scoring of the upper right first molars only.

Table 5 presents narrow-sense heritabilities for hypocone score variability in the sample. All values were high and ranged between 0.87 and 0.93 . The second molars had slightly higher estimates than the first molars.

\section{DISCUSSION}

In this study, the 6-grade scoring method developed by Turner et al. (1991) for classifying hypocone expression was shown to be relatively easy to use and to provide consistent results. The intra-observer reliability of $98 \%$ was slightly higher than that of Takahashi et al. (2007) who reported a concordance rate of $92 \%$ for
TABLE 5. Narrow-sense heritability estimates $\left(h^{2}\right)$ for hypocone expression of maxillary molars in Australian twins

\begin{tabular}{lccc}
\hline \multicolumn{1}{c}{ Tooth $^{1}$} & $\mathrm{~h}^{2}$ & $\mathrm{~L}_{1}$ & $\mathrm{~L}_{2}$ \\
\hline Right first molar & 0.87 & 0.65 & 0.96 \\
Left first molar & 0.87 & 0.67 & 0.97 \\
Right second molar & 0.90 & 0.80 & 0.95 \\
Left second molar & 0.93 & 0.86 & 0.97 \\
\hline
\end{tabular}

${ }^{1} \mathrm{~h}^{2}$ is heritability estimate; $\mathrm{L}_{1}$ is lower $95 \%$ confidence limit of the $\mathrm{h}^{2}$ estimate, and $\mathrm{L}_{2}$ is the upper $95 \%$ confidence limit.

scoring categories of hypocone expression on two separate occasions. The inter-observer assessment study showed a concordance of $70-74 \%$ which was similar to that found by Nichol and Turner (1986) who recorded concordance between observers of $70-75 \%$ for ranked traits (error rate $25-30 \%$ ).

The relative sizes of the cusps tended to correspond with phylogenetic and ontogenic timing of cusp formation. Apart from one case, the hypocone was shown to be reduced from the maxillary first to second molar. The one exception could possibly be due to different crown morphology making scoring difficult. The overall results were consistent with previous cusp area studies (Nichol and Turner, 1986; Macho and Moggi-Cecchi, 1992; Takahashi et al., 2007) and support the morphogenic field concept of Dahlberg (1945).

As reported by Takahashi et al. (2007) this study did not show any statistically significant difference in the occurrence of hypocones between males and females. However, this may be a reflection on the categorical system of classification used, which gives little information about actual size variation. This study did, however, show a tendency for higher frequencies of larger hypocone expressions in males than females. Kondo et al. (2005) also reported that larger distal cusps were found in males rather than females. Hence, it is

TABLE 4. Genetic model structure and associated statistics for hypocone scores on the maxillary right first molar ${ }^{1}$

\begin{tabular}{cccccc}
\hline Parameterization & $\mathrm{n}$ & Parameters & -2 Log Likelihood & $\mathrm{df}$ & AIC \\
\hline ACE & 176 & 7 & 255.9 & 170 & -84 \\
ADE & 176 & 7 & 256.0 & 170 & -84 \\
AE & 176 & 6 & 256.0 & 171 & -86 \\
CE & 176 & 6 & 260.8 & 171 & -81 \\
E & 176 & 5 & 286.1 & 172 & -58 \\
\hline
\end{tabular}

${ }^{1}$ Abbreviations: $\mathrm{n}=$ sample size; $\mathrm{df}=$ degrees of freedom; AIC = Akaike's Information Criterion 
likely that there is some influence of the sex chromosomes on the hypocone trait.

Hypocone expression was shown to be symmetrical between antimeric first molars except for one instance where the expression on the left was greater than the right, i.e. score 4 compared with score 1 . A number of researchers have suggested that both sides of the dental arch are under the influence of common genetic factors (Potter et al., 1976; Baume and Crawford, 1980). The findings of this study would support this hypothesis but the observed asymmetry in one individual would suggest environmental factors can lead to antimeric asymmetry.

The fact that this study shows a lower rate of concordance for hypocone expression between right and left maxillary second molars than first molars relates well to the schedule of tooth development and the theory that there is an association between early crown formation and low morphological variation of the first molar (Macho and Cecchi, 1992). These findings support the contention that certain teeth in the dentition, generally the earlier-developing members of each tooth class, are under stronger genetic control than laterforming teeth that are more subject to environmental influences (Corruccini and Potter, 1981).

It is assumed that MZ twins share $100 \%$ of their genes but the similarities between them can be due partly to shared pre- and post-natal environment (Scott and Turner, 1997). Common environment is perfectly correlated between twins in both zygosity groups whereas unique or non-shared environment only contributes to differences between twins. If genes are responsible for the expression of a trait, then a higher concordance of expression between MZ twin pairs would be expected compared with that seen between DZ twin pairs. This is what was noted in this study. However, although the concordance rate of expression of hypocones in MZ twin pairs was higher than that in DZ twin pairs, the concordance was not $100 \%$.

Narrow-sense heritability estimates indicate the proportion of the phenotypic variation attributable to additive genetic effects. Narrow-sense heritability is a measure of the degree to which individual phenotypes are determined by genes passed from parents to offspring, expressed as the ratio of the additive genetic variance to the total phenotypic variance. The high heritability estimates noted in this study suggest that most of the variation in expression of hypocones is due to genetic influences but environmental factors can still contribute to the observed variation. Hypocones were universally present on first molars; the second molars demonstrated a greater range of phenotypic expression than the first molars, with absence noted in some individuals. This may suggest that there is a common genetic liability for hypocone expression on both the first and second molars, which is modulated by differences in size, location and/or developmental timing events between these teeth.

In studies aimed at disclosing patterns in estimates of heritabilities, it has generally been assumed that the highest heritability will be displayed by the key tooth in each morphogenetic field (Townsend et al., 2008). This was not noted in this study; in fact, slightly higher values were achieved by the second molar compared with the first. It is considered that the longer a cusp remains in its soft tissue stage prior to mineralisation the more likely phenotypic variation will occur since odontogenesis involves a series of complicated epigenetic and morphogenic events. (Townsend et al., 2008). Due to the relatively small variation in hypocone size on the first molars, only three categories were analysed (score 3 and below, score 4 and score 5), whereas when looking at second molars all six categories were considered. This difference in the categories of expression analysed between the first and second molars may have influenced the heritability estimates, contributing to the lower scores noted for the first molars.

\section{CONCLUSIONS}

The hypotheses of this study were generally supported in that:

1. Hypocone expression was more common and larger in maxillary first molars than second molars.

2. Although sexual dimorphism was not statistically significant, there was a trend for males to have larger scores than females.

3. The expression of hypocones was symmetrical between antimeric teeth, with the concordance rates between sides being higher in first molars than second molars.

4. Monozygotic twin pairs exhibited a higher concordance rate hypocone expression than dizygotic twin pairs.

The results of model fitting and calculation of heritability estimates indicated that genetic factors exert a strong influence on hypocone expression in human maxillary molar teeth but environmental factors can also contribute to observed variance.

\section{ACKNOWLEDGEMENTS}

The authors would like to particularly acknowledge Dr Zaliha Ismail, who initiated this project but sadly was unable to see it through to publication. We also acknowledge the Minister for Police in South Australia and the South Australian Police. This study also forms part of an ongoing investigation of the teeth and faces of Australian twins and their families that is supported by the National Health and Medical Research Council of Australia and the Australian Dental Research Foundation. The authors would like to thank the twins and their families who have agreed to participate in this research project and the Australian Twin Registry for their continuing assistance. 


\section{LITERATURE CITED}

Baume RM, Crawford MH. 1980. Discrete dental trait asymmetry in Mexican and Brazilian groups. Am J Phys Anthropol 52:315-321.

Butler PM. 1939. Studies of the mammalian dentition. Differentiation of the post-canine dentition. Proc Zool Soc of London 109:1-36.

Butler PM. 1956.The ontogeny of molar pattern. Biol Rev 31:30-70.

Corruccini RS, Potter RHY. 1981. Developmental correlates of crown components asymmetry and occlusal discrepancy. Am J Phys Anthropol 55:21-31.

Dahlberg A. 1945. The changing dentition of man. J Am Dent Ass 32:676-690.

Dempsey PJ, Townsend GC, Richards LC. 1999. Increased tooth crown size in females with twin brothers: evidence for hormonal diffusion between twins in utero. Am J Hum Biol 11:577-586.

Falconer DS. 1965. The inheritance of liability to diseases estimated from the incidence among relatives. Ann Hum Genet 29:51-75.

Grayson DA. 1989. Twins reared together: minimizing shared environmental effects. Behav Genet 19:593604.

Hewitt JK. 1989. Of biases and more in the study of twins reared together: a reply to Grayson. Behav Genet 19:605-608.

Jernvall J. 2000. Linking development with generation of novelty in mammalian teeth. Proc Natl Acad Sci USA 97:2641-2645.

Jernvall J, Jung HS. 2000. Genotype, phenotype and developmental biology of molar tooth characters. Am J Phys Anthropol 31:171-190.

Jinks JL, Fulker DW. 1970. Comparison of the biometrical genetical, MAVA, and classical approaches to the analysis of human behaviour. Psychol Bull 73:311349.

Kabban M, Fearne J, Jovanovski V, Zou L. 2001. Tooth size and morphology in twins. Int J Paediatr Dent 11:333-339.

Kondo S, Townsend GC, Yamada H. 2005. Sexual dimorphism of cusp dimensions in human maxillary molars. Am J Phys Anthropol 128:870-877.

Macho GA, Moggi-Cecchi JM. 1992. Reduction of maxillary molars in Homo sapiens sapiens: a different perspective. Am J Phys Anthropol 87:151-159.
Mitsiadis TA, Smith MM. 2006. How do genes make teeth to order through development? J Exp Zool 306b:177-182.

Neale MC, Boker SM, Xie G, Maes HH. 2006. Mx: statistical modeling. VCU Box 900126, Richmond, VA 23298: Department of Psychiatry, 7th ed.

Neale MC, Cardon LR. 1992. Methodology for genetic studies of twins and families. Dordrecht, The Netherlands: Kluwer Academic Press.

Nichol CR, Turner CG II. 1986. Intra- and interobserver concordance in observing dental morphology. Am J Phys Anthropol 69:299-315.

Osborne JH 1978. Morphogenetic gradients: fields versus clones. In: Development, function and evolution of teeth. Butler PM and Joysey KA editors. New York: Academic Press. p 171-201.

Pearson K. 1901. Mathematical contributions to the theory of evolution. VII. On the correlation of characters not quantitatively measurable. Philos Trans R Soc Lond Ser A 195: 1-47.

Potter RH, Nance WE, Yu PI, Davis WB. 1976. A twin study of dental dimension. II. Independent genetic determinants. Am J Phys Anthropol 44:397-412.

Reich T, James JW, Morris CA. 1972. The use of multiple thresholds in determining the mode of transmission of semi-continuous traits. Ann Hum Genet Lond 36: 163-183.

Scott GR, Turner CG II.1997. The anthropology of human teeth. Cambridge: Cambridge University Press.

Takahashi M, Kondo S, Townsend G, Kanazawa E. 2007. Variability in cusp size of human maxillary molars, with particular reference to the hypocone. Arch Oral Biol 52:1146-1154.

Townsend G, Harris EF, Lesot H, Clauss F, Brook A. 2008. Morphogenetic fields within the human dentition: a new, clinically relevant synthesis of an old concept. Arch Oral Biol doi:10.1046/jarchoralbio.2008.06.011.

Turner CG II, Nichol CR, Scott GR. 1991. Scoring procedures for key morphological traits of the permanent dentition: the Arizona State University dental anthropology system. In: Advances in dental antropology. Kelly MA, Larsen CS, editors. New York: Wiley-Liss. p 13-31. 